\title{
Pseudo-Spectral Method for Space Fractional Diffusion Equation
}

\author{
Yiting Huang, Minling Zheng* \\ School of Science, Huzhou Teachers College, Huzhou, China \\ Email: 272277349@qq.com, ${ }^{*}$ mlzheng@aliyun.com
}

Received July 7, 2013; revised August 7, 2013; accepted August 15, 2013

Copyright (C) 2013 Yiting Huang, Minling Zheng. This is an open access article distributed under the Creative Commons Attribution License, which permits unrestricted use, distribution, and reproduction in any medium, provided the original work is properly cited.

\begin{abstract}
This paper presents a numerical scheme for space fractional diffusion equations (SFDEs) based on pseudo-spectral method. In this approach, using the Guass-Lobatto nodes, the unknown function is approximated by orthogonal polynomials or interpolation polynomials. Then, by using pseudo-spectral method, the SFDE is reduced to a system of ordinary differential equations for time variable $t$. The high order Runge-Kutta scheme can be used to solve the system. So, a high order numerical scheme is derived. Numerical examples illustrate that the results obtained by this method agree well with the analytical solutions.
\end{abstract}

Keywords: Riemann-Liouville Derivative; Pseudo-Spectral Method; Collocation Method; Fractional Diffusion Equation

\section{Introduction}

Anormalous diffusion model, where a particle spreads at a rate inconsistent with the classical Brownian motion model, has been applied in many fields, such as in fractured and porous media, in chaotic or turbulent processes [1-6]. It is known that anomalous diffusion processes can be described by fractional partial differential equations

$$
\frac{\partial u(x, t)}{\partial t}=\frac{\partial^{1-\gamma}}{\partial t^{1-\gamma}} \frac{\partial^{2} u(x, t)}{\partial x^{2}}+f(x, t), 0<\gamma<1
$$

or

$$
\frac{\partial u(x, t)}{\partial t}=\frac{\partial^{\alpha}}{\partial x^{\alpha}} u(x, t)+f(x, t), 1<\alpha<2
$$

where the fractional derivatives are defined as the Riemann-Liouville's representation. The former is referred as time fractional diffusion equation and the latter as space fractional diffusion equation. The difference between two cases can be shown using the interpolation of operator. It is well-known that the unknown $u(x, t)$ denotes the probability density function, which is the probability of finding the particle at position $x$ and at time $t$. For time fractional diffusion Equation (1.1), it

"Corresponding author. can be rewritten as (under suitable conditions)

$$
\frac{\partial^{\gamma} u(x, t)}{\partial t^{\gamma}}=\frac{\partial^{2} u(x, t)}{\partial x^{2}}+\tilde{f}(x, t), 0<\gamma<1
$$

where $\tilde{f}$ depends only on $f$. Hence, from the view point of mathematics, the Equation (1.3) can be thought as the interpolation of the equations in the case $\gamma=0$ and the case $\gamma=1$. In a word, the particles described by the Equation (1.3) are diffused slowly in comparison with the classic situations, namely sub-diffusion.

On the other hand, the fractional derivative of Equation (1.2) can similarly be thought as the interpolation of operators $\partial / \partial x$ and $\partial^{2} / \partial x^{2}$. Note that $\partial u / \partial x$ describes the free transport of particles and $\partial^{2} u / \partial x^{2}$, the diffusion, due to the collisions of particles. So, in the model of space fractional diffusion the velocity of particle is larger than one of ordinary diffusion, namely super-diffusion.

Several methods have been developed for numerical solving the fractional diffusion equation. Langlands and Henry studied sub-diffusion equation based on L1 scheme [7]. The authors proposed an implicit difference scheme which is unconditional stable. Based on Grünwald-Letnikov formula, an explicit difference scheme has been presented for sub-diffusion equation $[8,9]$.

In contrast to sub-diffusion equation, numerical solu- 
tion of supper-diffusion equations have been studied and some methods are also developed. It is interesting to note that the explicit and implicit difference schemes based on Grünwald-Letnikov formula are all unconditional unstable for super-diffusion Equation (1.2) and advectiondiffusion equation $[10,11]$. However, the authors proved that a shifted Grünwald-Letnikov formula can produce stable difference scheme [10,11].

The finite difference method is the most classic method for fractional differential equation [12-16]. The recent works can see the references [17-19]. However, high order accuracy schemes are seldom derived by finite difference method. In general, extrapolation method is applied in order to obtain a high accuracy $[10,16]$. It is well-known that spectral methods are superior to finite different methods in many instances for partial differential equations [20-24]. In the recent paper [25], the authors presented a spectral method to calculate the fractional derivative and integral, and studied the numerical solution of differential equations by the spectral method.

In this paper, we shall deal with the numerical solution of the one-dimensional variable coefficients space fractional diffusion equation

$$
\frac{\partial u(x, t)}{\partial t}=d(x) \frac{\partial^{\alpha}}{\partial x^{\alpha}} u(x, t)+q(x, t),(x, t) \in[0, L] \times(0, T]
$$

with the initial condition $u(x, 0)=h(x)$ for $0<x<L$ and boundary conditions $u(0, t)=0$ and $u(L, t)=g(t)$. Here, $d(x)$ is variable coefficient and $q(x, t)$ source term.

Chebyshev spectral collocation method (often called pseudo-spectral) is used in this paper. By employing the Chebyshev polynomials and Gauss-Lobbato nodes, the unknown is approximated by using the orthogonal projection and interpolation. So, the spatial fractional derivatives are easily computed and a system of ordinary differential equations in time can be derived. Then, high order Runge-Kutta method can be utilized and a high order accuracy scheme is obtained. In a comparison with the finite difference method, the Chebyshev spectral method used here shows remarkably superiority in terms of accuracy and the number of grid points required.

The paper is arranged as follows. The Section 2 introduces some basic concepts of fractional derivative and Jacobi orthogonal polynomials and their properties. The third section presents the spectral method for calculating the Riemann-Liouville fractional derivative. The pseudospectral method and its implement are proposed in Section 4. In Section 5, several numerical examples are provided. These numerical examples illustrate the high accuracy and efficiency of our method. Finally, we give the conclusion in Section 6.

\section{Preliminary}

\subsection{Fractional Derivative}

The Riemann-Liouville fractional integral of order $\alpha(\alpha>$ 0 ) for casual function $f(x)$ is defined by

$$
I^{\alpha} f(x)=\frac{1}{\Gamma(\alpha)} \int_{0}^{x} \mathrm{~d} x_{1} \int_{0}^{x_{1}} \mathrm{~d} x_{2} \cdots \int_{0}^{x_{n-1}} f\left(x_{n}\right) \mathrm{d} x_{n}
$$

where $\Gamma($.$) is the Gamma function. Riemann-Liouville$ fractional integral is an analogue of the well-known Cauchy formula, which reduces the $n$-fold integration

$$
\mathrm{I}^{n} f(x)=\int_{0}^{x} \mathrm{~d} x_{1} \int_{0}^{x_{1}} \mathrm{~d} x_{2} \cdots \int_{0}^{x_{n-1}} f\left(x_{n}\right) \mathrm{d} x_{n}
$$

into the Laplace convolution

$$
\mathrm{I}^{n} f(x)=\mathrm{K}_{n}(x) * f(x)=\int_{0}^{x} \mathrm{~K}_{n}(x-s) f(s) \mathrm{d} s
$$

where

$$
\mathrm{K}_{n}(x)=\frac{x^{n-1}}{(n-1) !} .
$$

For $\alpha=0, \mathrm{I}^{0} f(x)=f(x)$. It is easily obtained

$$
\mathrm{I}^{\alpha} x^{\mu}=\frac{\Gamma(\mu+1)}{\Gamma(\mu+1+\alpha)} x^{\mu+\alpha},
$$

for $\alpha \geq 0, \mu>-1$.

The Riemann-Liouville representation of fractional derivative of order $\alpha(\alpha \geq 0)$ for $f(x)$ is defined by

$$
\begin{aligned}
D^{\alpha} f(x) & =D^{m} \circ \mathrm{I}^{\alpha} f(x) \\
& =\frac{1}{\Gamma(m-a)} \frac{\mathrm{d}^{m}}{\mathrm{~d} x^{m}} \int_{0}^{x}(x-s)^{m-\alpha-1} f(s) \mathrm{d} s
\end{aligned}
$$

here $m-1<\alpha<m$.

By the part integration formula, one can easily derive the following properties.

Proposition 2.1. Let $m-1 \leq \alpha<m, m \in N$, then

$$
\begin{aligned}
& D^{\alpha} f(x)=\sum_{k=0}^{m-1} \frac{f^{(k)}(0)}{\Gamma(k+1-\alpha)} x^{k-\alpha} \\
& +\frac{1}{\Gamma(m-\alpha)} \int_{0}^{x}(x-\xi)^{m-\alpha-1} f^{(m)}(\xi) \mathrm{d} \xi .
\end{aligned}
$$

Proposition 2.2. Let $\mu>-1, \alpha \geq 0$, then

$$
D^{\alpha} x^{\mu}=\frac{\Gamma(\mu+1)}{\Gamma(\mu-\alpha+1)} x^{\mu-\alpha}
$$

\subsection{Jacobi Orthogonal Polynomials}

Given a weight function $\omega(x)$, the orthogonal polynomials sequence $\left.\left\{P_{j}(x)\right\}\right|_{j=0} ^{\infty}$, with $\operatorname{deg}\left(P_{j}\right)=j$ can be written into [26]

$$
P_{0}(x)=1, P_{1}(x)=x-\xi_{1},
$$




$$
P_{n+1}(x)=\left(x-\xi_{n+1}\right) P_{n}(x)-\zeta_{n+1} P_{n-1}(x), n \geq 1
$$

in which

$$
\begin{gathered}
\xi_{n+1}=\frac{\left(x P_{n}, P_{n}\right)_{\omega}}{\left(P_{n}, P_{n}\right)_{\omega}}, n \geq 0, \\
\zeta_{n+1}=\frac{\left(x P_{n-1}, P_{n}\right)_{\omega}}{\left(P_{n-1}, P_{n-1}\right)_{\omega}}, n \geq 1
\end{gathered}
$$

here $(\cdot, \cdot)_{\omega}$ denotes weighted inner product of a Hilbert space.

For $\omega(x)=(1-x)^{a}(1+x)^{b}, a, b>-1, x \in[-1,1]$, the Jacobi polynomials is a orthogonal polynomials sequence $\left.\left\{J_{n}^{a, b}(x)\right\}\right\}_{n=0}^{\infty}$ with

$$
\left\{\begin{array}{l}
J_{0}^{a, b}(x)=1, J_{1}^{a, b}=\frac{1}{2}(a+b+2) x+\frac{1}{2}(a-b), \\
J_{n+1}^{a, b}(x)=\left(A_{n}^{a, b} x-B_{n}^{a, b}\right) J_{n}^{a, b}(x)-C_{n}^{a, b} J_{n-1}^{a, b}(x), n \geq 1
\end{array}\right.
$$

where

$$
\begin{gathered}
A_{n}^{a, b}=\frac{(2 n+a+b+1)(2 n+a+b+2)}{2(n+1)(n+a+b+1)} \\
B_{n}^{a, b}=\frac{\left(b^{2}-a^{2}\right)(2 n+a+b+1)}{2(n+1)(n+a+b+1)(2 n+a+b)} \\
C_{n}^{a, b}=\frac{(n+a)(n+b)(2 n+a+b+2)}{(n+1)(n+a+b+1)(2 n+a+b)}
\end{gathered}
$$

The following is some useful properties of Jacobi polynomials that will be used in present paper [26] (also refer to [25] and references therein).

Proposition 2.3. 1) High order derivative for Jacobi polynomials

$$
\frac{\mathrm{d}^{m}}{\mathrm{~d} x^{m}} J_{n}^{a, b}(x)=c_{n, m}^{a, b} J_{n-m}^{a+m, b+m}(x), n \geq m, m \in N
$$

where

$$
c_{n, m}^{a, b}=\frac{\Gamma(n+m+a+b+1)}{2^{m} \Gamma(n+a+b+1)} .
$$

2) Expression by the derivative of Jacobi polynomials

$$
\begin{aligned}
J_{n}^{a, b}(x)= & \alpha_{n}^{a, b} \frac{\mathrm{d}}{\mathrm{d} x} J_{n-1}^{a, b}(x)+\beta_{n}^{a, b} \frac{\mathrm{d}}{\mathrm{d} x} J_{n}^{a, b}(x) \\
& +\gamma_{n}^{a, b} \frac{\mathrm{d}}{\mathrm{d} x} J_{n+1}^{a, b}(x), n \geq 1
\end{aligned}
$$

where

$$
\begin{aligned}
\alpha_{1}^{a, b} & =0, \\
\alpha_{n}^{a, b} & =\frac{-2(n+a)(n+b)}{(n+a+b)(2 n+a+b)(2 n+a+b+1)}(n \geq 2) \\
\beta_{n}^{a, b} & =\frac{2(a-b)}{(2 n+a+b)(2 n+a+b+2)} \\
\gamma_{n}^{a, b} & =\frac{2(n+a+b+1)}{(2 n+a+b+1)(2 n+a+b+2)}
\end{aligned}
$$

By the previous Jacobi polynomials some special orthogonal polynomials can be obtained. Legendre and Chebyshev polynomials are two important polynomials of the special cases of the Jacobi polynomials. For the case of $a=b=0, \omega(x)=1$, the corresponding polynomials is said to Legendre polynomials; and the case of $a=b=-\frac{1}{2}, \omega(x)=\frac{1}{\sqrt{1-x^{2}}}$ corresponds to Chebyshev polynomials.

\section{Spectral Approximation to Fractional Derivative}

Let $H_{N}$ denote the set of polynomials of degree not exceeding $N$. It is clear that

$$
H_{N}=\operatorname{span}\left\{J_{0}^{a, b}(x), J_{1}^{a, b}(x), \cdots, J_{N}^{a, b}(x)\right\}
$$

Denote the weighted inner product for weight function $\omega(x)$ on interval $I=[-1,1]$

$$
(u, v)_{\omega}=\int_{I} u(x) v(x) \omega(x) \mathrm{d} x
$$

and the weighted Lebesgue space

$$
L_{\omega}^{2}(I)=\left\{f \mid(f, f)_{\omega}<+\infty\right\}
$$

with norm $\|u\|_{L_{\omega}^{2}}=(u, u)_{\omega}^{\frac{1}{2}}$.

Define the orthogonal projection

$$
\Pi_{N}: L_{\omega}^{2}(I) \rightarrow H_{N}
$$

Then function $u(x) \in L_{w}^{2}(I)$ can be approximated by the orthogonal projection

$$
u_{N}(x)=\Pi_{N} u(x)=\sum_{n=0}^{N} \hat{u}_{n}^{a, b} J_{n}^{a, b}(x)
$$

The expression coefficients $\hat{u}_{n}^{a, b}$ is determined by

$$
\hat{u}_{n}^{a, b}=\left(u, J_{n}^{a, b}\right)_{w} /\left(J_{n}^{a, b}, J_{n}^{a, b}\right)_{w} .
$$

Therefore, the fractional derivative $D^{\alpha} u(x)$ can be approximated by

$$
D^{\alpha} u_{N}(x)=\sum_{n=0}^{N} \hat{u}_{n}^{a, b} d_{n}^{a, b, \alpha}(x), d_{n}^{a, b, \alpha}(x)=D^{\alpha} J_{n}^{a, b}(x)
$$

where 


$$
D^{\alpha} J_{n}^{a, b}(x)=\frac{1}{\Gamma(m-\alpha)} \frac{\mathrm{d}^{m}}{\mathrm{~d} x^{m}} \int_{-1}^{x}(x-\xi)^{m-\alpha-1} J_{n}^{a, b}(\xi) \mathrm{d} \xi
$$

Now, we consider the calculation of $d_{n}^{a, b, \alpha}(x)$. Let

$$
\hat{d}_{n}^{a, b, \alpha}(x)=\frac{1}{\Gamma(\alpha)} \int_{-1}^{x}(x-\xi)^{\alpha-1} J_{n}^{a, b}(\xi) \mathrm{d} \xi,
$$

Then, $\hat{d}_{n}^{a, b, \alpha}(x)$ can be the computed by recurrence formula [25]. Clearly,

$$
\begin{gathered}
\hat{d}_{0}^{a, b, \alpha}(x)=\frac{(x+1)^{\alpha}}{\Gamma(1+\alpha)}, \\
\hat{d}_{1}^{a, b, \alpha}(x)=\frac{a+b+2}{2}\left(\frac{(x+1)^{1+\alpha}}{\Gamma(2+\alpha)}-\frac{(x+1)^{\alpha}}{\Gamma(1+\alpha)}\right) \\
+\frac{a-b}{2} \hat{d}_{0}^{a, b, \alpha}(x)
\end{gathered}
$$

By the three-term recurrence relation (2.5),

$$
\begin{aligned}
\hat{d}_{n+1}^{a, b, \alpha}(x)= & \frac{A_{n}^{a, b}}{\Gamma(\alpha)} \int_{-1}^{x}(x-\xi)^{\alpha-1} \xi J_{n}^{a, b}(\xi) \mathrm{d} \xi \\
& -B_{n}^{a, b} \hat{d}_{n}^{a, b, \alpha}(x)-C_{n}^{a, b} \hat{d}_{n-1}^{a, b, \alpha}(x)
\end{aligned}
$$

Note that

$$
\begin{aligned}
& \frac{1}{\Gamma(\alpha)} \int_{-1}^{x}(x-\xi)^{\alpha-1} \xi J_{n}^{a, b}(\xi) \mathrm{d} \xi \\
= & \frac{1}{\Gamma(\alpha)} \int_{-1}^{x} x(x-\xi)^{\alpha-1} J_{n}^{a, b}(\xi) \mathrm{d} \xi \\
& -\frac{1}{\Gamma(\alpha)} \int_{-1}^{x}(x-\xi)^{\alpha} J_{n}^{a, b}(\xi) \mathrm{d} \xi .
\end{aligned}
$$

In light of (2.10) and notice that

$$
J_{n}^{a, b}(-1)=(-1)^{n} \frac{\Gamma(n+b+1)}{n ! \Gamma(b+1)},
$$

one can obtain

$$
\begin{aligned}
& \frac{1}{\Gamma(\alpha)} \int_{-1}^{x}(x-\xi)^{\alpha} J_{n}^{a, b}(\xi) \mathrm{d} \xi \\
= & \frac{1}{\Gamma(\alpha)} \int_{-1}^{x}(x-\xi)^{\alpha}\left(\alpha_{n}^{a, b} \frac{\mathrm{d}}{\mathrm{d} \xi} J_{n-1}^{a, b}(\xi)\right. \\
& \left.+\beta_{n}^{a, b} \frac{\mathrm{d}}{\mathrm{d} \xi} J_{n}^{a, b}(\xi)+\gamma_{n}^{a, b} \frac{\mathrm{d}}{\mathrm{d} \xi} J_{n+1}^{a, b}(\xi)\right) \mathrm{d} \xi \\
= & \frac{(-1)^{n}(x+1)^{\alpha}}{\Gamma(\alpha) \Gamma(b+1)}\left[\frac{\Gamma(n+b) \alpha_{n}^{\alpha, b}}{(n-1) !}\right. \\
& \left.-\frac{\Gamma(n+b+1) \beta_{n}^{\alpha, b}}{n !}+\frac{\Gamma(n+b+2) \gamma_{n}^{\alpha, b}}{(n+1) !}\right] \\
& +\alpha\left[\alpha_{n}^{a, b} \hat{d}_{n-1}^{a, b, \alpha}(x)+\beta_{n}^{a, b} \hat{d}_{n}^{a, b, \alpha}(x)+\gamma_{n}^{a, b} \hat{d}_{n+1}^{a, b, \alpha}(x)\right]
\end{aligned}
$$

Thus, $\hat{d}_{n+1}^{a, b, \alpha}(x)$ can be derived

$$
\begin{aligned}
& \hat{d}_{n+1}^{a, b, \alpha}(x)=-\frac{\alpha \alpha_{n}^{a, b} A_{n}^{a, b}+C_{n}^{a, b}}{1+\alpha \gamma_{n}^{a, b} A_{n}^{a, b}} \hat{d}_{n-1}^{a, b, \alpha}(x) \\
& +\frac{\left(x-\alpha \beta_{n}^{a, b}\right) A_{n}^{a, b}-B_{n}^{a, b}}{1+\alpha \gamma_{n}^{a, b} A_{n}^{a, b}} \hat{d}_{n}^{a, b, \alpha}(x) \\
& -\frac{(-1)^{n} A_{n}^{a, b} z_{n}^{a, b, \alpha}(x+1)^{\alpha}}{1+\alpha \gamma_{n}^{a, b} A_{n}^{a, b}}
\end{aligned}
$$

in which

$$
\begin{aligned}
& z_{n}^{a, b, \alpha}=\frac{\Gamma(n+b)}{\Gamma(n+1) \Gamma(a)(n-1) !} \alpha_{n}^{a, b} \\
& -\frac{\Gamma(n+b+1)}{\Gamma(n+1) \Gamma(a) n !} \beta_{n}^{a, b}+\frac{\Gamma(n+b+2)}{\Gamma(n+1) \Gamma(a)(n+1) !} \gamma_{n}^{a, b}
\end{aligned}
$$

and $A_{n}^{a, b}, B_{n}^{a, b}, C_{n}^{a, b}$ is defined as (2.6)-(2.8), $\alpha_{n}^{a, b}$, $\beta_{n}^{a, b} \gamma_{n}^{a, b}$ as (2.11). Therefore, we obtain by (2.3) and (2.9) that

$$
\begin{aligned}
& d_{n}^{a, b, \alpha}(x)=\sum_{k=0}^{m-1} \frac{\mathrm{d}^{k}}{\frac{\mathrm{d} x^{k}}{\Gamma(k+1-\alpha)}} J_{n}^{a, b}(-1) \\
& +\frac{1}{\Gamma(m-\alpha)} \int_{-1}^{x}(x-\xi)^{m-1-\alpha} \frac{\mathrm{d}^{m}}{\mathrm{~d} \xi^{m}} J_{n}^{a, b}(\xi) \mathrm{d} \xi \\
& =\sum_{k=0}^{m-1} \frac{(-1)^{n-k} c_{n, k}^{a, b} \Gamma(n+b+1)}{\Gamma(k+1-\alpha) \Gamma(b+k+1)(n-k) !}(x+1)^{k-\alpha} \\
& +c_{n, m}^{a, b} \hat{d}_{n-m}^{a+m, b+m, m-\alpha}(x)
\end{aligned}
$$

Remark 1. By the standard theory of orthogonal projection, the spectral accuracy to approximate the fractional derivative $D^{\alpha} u(x)$ can be obtained (refer to the references $[25,26])$.

\section{Collocation Method}

This section presents the Chebyshev collocation method (often called pseudo-spectral method) for solving the space fractional diffusion Equation (1.4). Firstly, we shall consider the Gauss-Lobatto nodes in order to obtain the collocation equation.

Consider the Chebyshev polynomials $T_{n}(x)$ of the first kind, which are the special case of Jacobi polynomials

$$
T_{n}(x)=\frac{2^{2 n}(n !)^{2}}{(2 n) !} J_{n}^{-\frac{1}{2},-\frac{1}{2}}(x), n=0,1,2, \cdots
$$

for $-1 \leq x \leq 1$. Chebyshev polynomials are the weighted orthogonal polynomials with weight function

$$
w(x)=\frac{1}{\sqrt{1-x^{2}}} .
$$


One can easily obtain the three-term recurrence formula

$$
\begin{aligned}
& T_{0}(x)=1, T_{1}(x)=x, \\
& T_{k+1}(x)=2 x T_{k}(x)-T_{k-1}(x), k=1,2, \cdots
\end{aligned}
$$

Denote $Q(x)=T_{N+1}(x)+p T_{N}(x)+q T_{N-1}(x)$, here $p$, $q$ is determined by satisfying $Q( \pm 1)=0$. Then the roots of $Q(x)=0$ is named as the Chebyshev Gauss-Lobatto points (in this paper is also called Gauss-Lobatto points). Gauss-Lobatto points can be explicitly written as $[26,27]$

$$
x_{k}=\cos \frac{k \pi}{N}, K=0,1,2, \cdots, N .
$$

Define the discrete inner product $(\because)_{N}$ as

$$
(u(x), v(x))_{N}=\sum_{k=0}^{N} u\left(x_{k}\right), v\left(x_{k}\right) \omega_{k}
$$

where $\omega_{k}$ are the associated weights of Chebyshev Gauss-Lobatto integration

$$
\omega_{k}=\frac{\pi}{c_{k} N}, c_{k}=\left\{\begin{array}{l}
2 \text { for } k=0, N, \\
1 \text { for } k=1, \cdots, N-1 .
\end{array}\right.
$$

By orthogonality it can easily be derived that

$$
\sum_{k=0}^{N} T_{i}\left(x_{k}\right) T_{j}\left(x_{k}\right) \omega_{k} \text {, if } i \neq j \leq N
$$

Next, let us consider the following Gauss-Lobatto points

$$
x_{k}=\frac{L}{2}+\frac{L}{2} \cos \frac{k \pi}{N}, K=0,1,2, \cdots, N .
$$

Assume that the approximate solution of the space fractional diffusion Equation (1.4) has separable formation

$$
u(x, t) \approx u_{N}(x, t)=\sum_{n=0}^{N} \phi(t) T_{n}(\hat{x}), \quad \hat{x}=\frac{2 x}{L}-1
$$

for $x \in[0, L]$. Replacing $u(x, t)$ in (1.4) by $u_{N}(x, t)$ it results in the following equations on $x_{k}$

$$
\left\{\begin{array}{l}
\frac{\partial}{\partial t} u_{N}\left(x_{k}, t\right)=d\left(x_{k}\right) \frac{\partial^{\alpha}}{\partial x^{\alpha}} u_{N}\left(x_{k}, t\right)+q\left(x_{k}, t\right), \\
k=1,2, \cdots, N-1 \\
u_{N}\left(x_{N}, t\right)=0, u_{N}\left(x_{0}, t\right)=g(t)
\end{array}\right.
$$

with initial condition $u_{N}(x, 0)=h(x)$, where $1<\alpha<2$. Set $\hat{x}_{k}=\frac{2 x_{k}}{L}-1$, then Equations (4.4) can be written into

$$
\begin{aligned}
& \sum_{n=0}^{N} T_{n}\left(\hat{x}_{k}\right) \frac{\mathrm{d}}{\mathrm{d} t} \phi_{n}(t) \\
= & \left.\sum_{n=0}^{N} d\left(x_{k}\right) D^{\alpha} T_{n}\left(\hat{x}_{k}\right)\right|_{\hat{x}=\hat{x}_{k}} \phi_{n}(t)+q\left(x_{k}, t\right),
\end{aligned}
$$

for $k=1,2, \cdots, N-1$. In addition, the boundary conditions for $x_{0}$ and $x_{N}$ can be expressed by

$$
\sum_{n=0}^{N} \phi_{n}(t) T_{n}\left(\hat{x}_{N}\right)=0, \sum_{n=0}^{N} \phi_{n}(t) T_{n}\left(\hat{x}_{0}\right)=g(t)
$$

Equations (4.5) and (4.6) is called the collocation equation for fractional diffusion Equation (1.4), and $x_{k}$ defined by (4.3) is called the collocation points.

The Equation (4.5) is a system of ordinary differential equations. The calculation of $D^{\alpha} T_{n}(x)$ can be implemented by

$$
\begin{aligned}
& D^{\alpha} T_{n}(\hat{x})=\frac{1}{\Gamma(2-\alpha)} \frac{\mathrm{d}^{2}}{\mathrm{~d} x^{2}} \int_{0}^{x}(x-\xi)^{1-\alpha} T_{n}(\hat{\xi}) \mathrm{d} \xi \\
& =\left(\frac{L}{2}\right)^{-\alpha} \frac{1}{\Gamma(2-\alpha)} \frac{\mathrm{d}^{2}}{\mathrm{~d} \hat{x}^{2}} \int_{-1}^{\hat{x}}(\hat{x}-\hat{\xi})^{1-\alpha} T_{n}(\hat{\xi}) \mathrm{d} \xi \\
& =\left(\frac{L}{2}\right)^{-\alpha} \frac{2^{2 n}(n !)^{2}}{(2 n) !} d_{n}^{a, b, \alpha}(\hat{x})
\end{aligned}
$$

here $\hat{\xi}=(2 \xi-l) / L, a=b=-1 / 2$, therefore $D^{\alpha} T_{n}\left(x_{k}\right)$ can be conveniently calculated by making use of (3.4).

Remark 2. 1) Equations (4.5) and (4.6) is also said to be the strong form of the collocation method.

2) In order to get high order schemes, high order Runge-kutta methods can be used to solve the system of ordinary differential Equation (4.5).

Now, we employ the discrete inner product to deal with the initial condition. Notice that

$$
u_{N}(x, 0)=h(x), 0<x<L,
$$

can be written into

$$
\sum_{n=0}^{N} \phi_{n}(0) T_{n}(\hat{x})=h(\hat{x}), \hat{x}=\frac{2 x}{L}-1
$$

Therefore, making use of (4.2) it gives

$$
\phi_{k}(0)=\frac{\left(h, T_{k}\right)_{N}}{\left(T_{k}, T_{k}\right)_{N}}, k=1,2, \cdots, N-1 .
$$

In order to conveniently deal with the boundary conditions and initial condition, let us consider another form of the collocation method based on interpolation. Based on the Gauss-Lobatto nodes (4.3) above, the Lagrangian interpolation basis function $\varphi_{k}(x)$ are given by

$$
\varphi_{k}(x)=\prod_{j \neq k} \frac{x-x_{j}}{x_{k}-x_{j}}, k=0,1, \cdots, N .
$$

Assume that the approximation $u_{N}(x, t)$ be the interpolation

$$
u_{N}(x, t)=\sum_{j=0}^{N} \phi_{j}(t) \varphi_{j}(x)
$$

Note that $\phi_{j}\left(x_{i}\right)=\delta_{j i}$, here $\delta_{j i}$ denotes the Kronecker 
delta symbol. Then, the Equations (4.6) simply become

$$
\phi_{0}(t)=g(t), \phi_{N}(t)=0,
$$

and the initial condition (4.7) is altered by

$$
\phi_{j}(0)=h\left(x_{j}\right), j=1,2, \cdots, N-1,
$$

Let

$$
\Phi(t)=\left(\phi_{1}\left((t), \phi_{2}(t), \cdots, \phi_{N-1}(t)\right)^{\mathrm{T}} .\right.
$$

Equation (4.5) is correspondingly modified into

$$
\Phi^{\prime}(t)=D M \Phi(t)+N g(t)+Q(t)
$$

where

$$
\begin{gathered}
M=\left(\begin{array}{cccc}
D^{\alpha} \varphi_{1}\left(X_{1}\right) & D^{\alpha} \varphi_{2}\left(X_{1}\right) & \cdots & D^{\alpha} \varphi_{N-1}\left(x_{1}\right) \\
D^{\alpha} \varphi_{1}\left(x_{2}\right) & D^{\alpha} \varphi_{2}\left(x_{2}\right) & \cdots & D^{\alpha} \varphi_{N-1}\left(X_{2}\right) \\
\vdots & \vdots & \ddots & \vdots \\
D^{\alpha} \varphi_{1}\left(x_{N-1}\right) & D^{\alpha} \varphi_{2}\left(X_{N-1}\right) & \cdots & D^{\alpha} \varphi_{N-1}\left(X_{N-1}\right)
\end{array}\right) \\
N=\left(\begin{array}{c}
d_{1} D^{\alpha} \varphi_{0}\left(x_{1}\right) \\
d_{2} D^{\alpha} \varphi_{0}\left(x_{2}\right) \\
\vdots \\
d_{N-1} D^{\alpha} \varphi_{0}\left(x_{N-1}\right)
\end{array}\right) Q(t)=\left(\begin{array}{c}
q_{1}(t) \\
q_{2}(t) \\
\vdots \\
q_{N-1}(t)
\end{array}\right)
\end{gathered}
$$

and

$$
D=\operatorname{diag}\left(d_{1}, d_{2}, \cdots, d_{N-1}\right),
$$

with $d_{i}=d\left(x_{i}\right), q_{i}(t)=q\left(x_{i}, t\right)$ for $1<i<N-1$.

In order to compute conveniently the coefficient matrix $M$, we expanse $\varphi_{k}^{\prime \prime}(x)$ into Taylor series

$$
\varphi_{k}^{\prime \prime}(x)=\varphi_{k}^{\prime \prime}(0)+\varphi_{k}^{\prime \prime \prime}(0) x+\cdots+\frac{1}{(N-2) !} \varphi_{k}^{(N)}(0) x^{N-2} .
$$

Therefore, making use of Prop. 2.1 and Prop. 2.2 one can derive.

$$
D^{\alpha} \varphi_{j}(x)=\sum_{m=0}^{N} \frac{\varphi_{j}^{(m)}(0)}{\Gamma(m+1-\alpha)} x^{m-\alpha},
$$

for $0 \leq x \leq 1$ and $0 \leq j \leq N$.

\section{Numerical Examples}

In this section, we consider the space fractional diffusion equations for different source terms and the values of $\alpha$ utilizing the collocation method. Here, the fourth order Runge-Kutta method is used for all the examples with time step $\Delta t=0.05$. The first example is a fractional equation with $1<\alpha<1.5$ and time variable belongs to $(0,+\infty)$. The second example is associated to the problem of time belonging to finite interval with $1.5<\alpha<2$. For the first example, we use the second kind of collocation method, namely based on the Lagrange interpolation, with $N=3$. The second example is solve by the Chebyshev polynomials approximation with $N=3$.

Example 1. Consider the following asymptotic problem with $\alpha=1.2$

$$
\begin{gathered}
\partial_{t} u(x, t)=d(x) \frac{\partial^{1.2}}{\partial x^{1.2}} u(x, t)+q(x, t), \\
(x, t) \in(0,1) \times(0,+\infty)
\end{gathered}
$$

with the initial condition

$$
u(x, 0)=x^{2}, x \in(0,1)
$$

and boundary conditions

$$
u(0, t)=0, u(1, t)=\mathrm{e}^{-t}, t \in(0,+\propto)
$$

in which

$$
d(x)=\frac{\Gamma(1.8)}{2} x^{2.2},
$$

and

$$
q(x, t)=-x^{2}(x+1) \mathrm{e}^{-t} .
$$

The exact solution (Ex-solution) is $u(x, t)=x^{2} \mathrm{e}^{-t}$. The numerical solution (CM-solution) at time $t=1$, using the collocation method based on the Lagrange interpolation, is shown in Figure 1.

Example 2. Consider the following finite interval problem with $\alpha=1.8$

$$
\begin{aligned}
& \partial_{t} u(x, t) \\
= & d(x) \frac{\partial^{1.8}}{\partial x^{1.8}} u(x, t)+q(x, t),(x, t) \in(0,1) \times(0, T]
\end{aligned}
$$

with the initial condition

$$
u(x, 0)=x^{2}, x \in(0,1)
$$

and boundary conditions

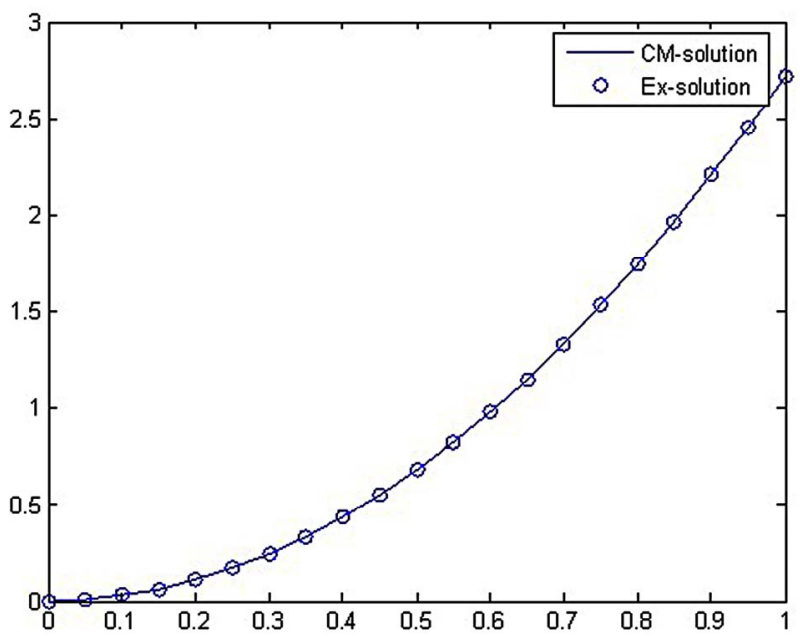

Figure 1. The exact solution and collocation solution for asymptotic problem with $\alpha=1.2$ at time $t=1$. 


$$
u(0, t)=0, u(1, t)=\mathrm{e}^{-t}, t \in(0, T]
$$

where

$$
\begin{gathered}
d(x)=\frac{\Gamma(1.2)}{2} x^{0.8} \\
q(x, t)=x(x-1) \mathrm{e}^{t} .
\end{gathered}
$$

The exact solution is $u(x, t)=x^{2} \mathrm{e}^{t}$. The numerical solution at time $t=1$, using the collocation method based on the Chebyshev polynomials with $N=3$, is shown in Figure 2. The error err $=\left|u-u_{N}\right|$ is illustrated in Table 1.

\section{Conclusion}

The collocation method, namely called pseudo-spectral method, is proposed in present paper. This kind of method can be efficiently applied to fractional partial differential equations. The remarkably superiorities, efficiency and high accuracy have been found through the numerical examples presented in this paper. The high accurate approximation only by a few grids can be derived. However, the space and time steps must satisfy certain condition in order to guarantee the stability for finite difference method. It must be stressed that the higher order collocation method based on Lagrange interpolation maybe result in numerical instability because of the ill-condition of the matrix $D^{\alpha} \varphi_{k}\left(x_{l}\right)$. It is hoped that the error estimated on the spectral method for fractional differential equations will be studied in future work.

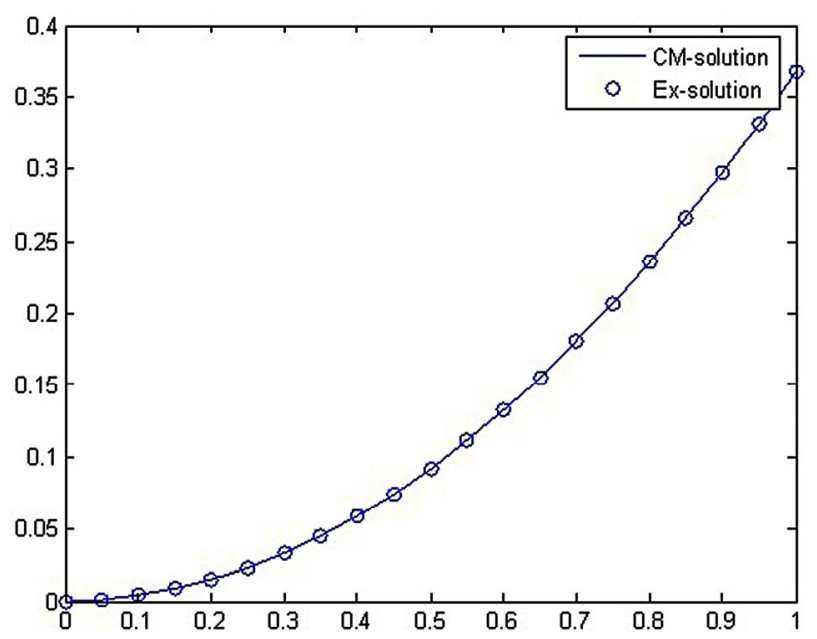

Figure 2. The exact solution and collocation solution for finite interval problem with $\alpha=1.8$ at time $t=1$.

Table 1. The error of the collocation solution with $N=3$.

\begin{tabular}{cccccc}
\hline$x$ & 0.1 & 0.2 & 0.3 & 0.4 & 0.5 \\
\hline err & $0.2465 \mathrm{e}-05$ & $0.3628 \mathrm{e}-05$ & $0.3771 \mathrm{e}-05$ & $0.3178 \mathrm{e}-05$ & $0.2131 \mathrm{e}-05$ \\
\hline$x$ & 0.6 & 0.7 & 0.8 & 0.9 & 1.0 \\
\hline err & $0.9139 \mathrm{e}-06$ & $0.1909 \mathrm{e}-06$ & $0.9002 \mathrm{e}-06$ & $0.9309 \mathrm{e}-06$ & 0 \\
\hline
\end{tabular}

\section{Acknowledgements}

The present paper was supported by the Natural Science Foundation of China (Grant No. 11101140) and the Nation Natural Science Foundation of Huzhou.

\section{REFERENCES}

[1] M. Giona and H. E. Roman, "Fractional Diffusion Equation for Transport Phenomena in Random Media," Journal of Physics A, Vol. 185, No. 1-4, 1992, pp. 87-97.

[2] R. Metzler, J. Klafter and I. M. Sokolov, "Anomalous Transport in External Fields: Continuous Time Random Walks and Fractional Diffusion Equations Extends," Physical Review E, Vol. 58, No. 3, 1998, pp. 1621-1633. http://dx.doi.org/10.1103/PhysRevE.58.1621

[3] R. Metzler and J. Klafter, "Boundary Value Problems for Fractional Diffusion Equations," Journal of Physics A, Vol. 278, No. 1-2, 2000, pp. 107-125.

[4] B. I. Henry and S. L. Wearne, "Fractional Reaction-Diffusion," Journal of Physics A, Vol. 276, No. 3-4, 2000, pp. 448-445.

[5] Y. Zhang, M. Meerschaert and B. Baeumer, "Particle Tracking for Time-Fractional Diffusion," Physical Review E, Vol. 78, No. 3, 2008, Article ID: 036705.

[6] H. G. Sun, W. Chen and Y. Q. Chen, "Variable-Order Fractional Differential Operators in Anomalous Diffusion Modeling," Journal of Physics A, Vol. 338, No. 21, 2009, pp. 4586-4592.

[7] A. T. M. Langlands and B. I. Henry, "The Accuracy and Stability of an Implicit Solution Method for the Fractional Diffusion Equation," Journal of Computational Physics, Vol. 205, No. 2, 2005, pp. 719-736. http://dx.doi.org/10.1016/j.jcp.2004.11.025

[8] S. B. Yuste and L. Acedo, "On an Explicit Finite Difference Method for Fractional Diffusion Equations," SIAM Journal on Numerical Analysis, Vol. 42, No. 5, 2005, pp. 1862-1874. http://dx.doi.org/10.1137/030602666

[9] S. B. Yuste, "Weighted Average Finite Difference Methods for Fractional Diffusion Equations," Journal of Computational Physics, Vol. 216, No. 1, 2006, pp. 264-274. http://dx.doi.org/10.1016/j.jcp.2005.12.006

[10] C. Tadjeran, M. M. Meerschaert and H.-P. Scheffler, "A Second Order Accurate Numerical Approximation for the Fractional Diffusion Equation," Journal of Computational Physics, Vol. 213, No. 1, 2006, pp. 205-213. http://dx.doi.org/10.1016/j.jcp.2005.08.008

[11] M. M. Meerschaert and C. Tadjeran, "Finite Difference Approximations for Fractional Advection-Dispersion Flow Equations," Journal of Computational and Applied Mathematics, Vol. 172, No. 1, 2004, pp. 65-77. http://dx.doi.org/10.1016/j.cam.2004.01.033

[12] L. Blank, "Numerical Treatment of Differential Equations of Fractional Order," Numerical Analysis Report 287, Manchester Centre for Computational Mathematics, Manchester, 1996.

[13] K. Diethelm and G. Walz, "Numerical Solution of Fractional Order Differential Equations by Extroplation," $\mathrm{Nu}$ - 
merical Algorithms, Vol. 16, 1997, pp. 231-253. http://dx.doi.org/10.1023/A:1019147432240

[14] K. Diethelm, "An Algorithm for the Numerical Solution of Differential Equations of Fractional Order," Electronic Transactions on Numerical Analysis, Vol. 5, 1997, pp. 1-6.

[15] N. Ford and A. Simpson, "The Numerical Solution of Fractional Differential Equations: Speed versus Accuracy," Numerical Analysis Report 385, Manchester Centre for Computational Mathematics, Manchester, 2001.

[16] K. Diethelm, N. Ford and A. Freed, "A Predictor-Corrector Approach for the Numerical Solution of Fractional Differential Equations," Nonlinear Dynamics, Vol. 29, No. 1-4, 2002, pp. 3-22. http://dx.doi.org/10.1023/A:1016592219341

[17] C.-M. Chen, F. Liu and K. Burrage, "Finite Difference Methods and a Fourier Analysis for the Fractional Reaction-Subdiffusion Equation," Applied Mathematics and Computation, Vol. 198, No. 2, 2008, pp. 754-769. http://dx.doi.org/10.1016/j.amc.2007.09.020

[18] B. Baeumer, M. Kovacs and M. M. Meerschaert, "Numerical Solutions for Fractional Reaction-Diffusion Equations," Computers \& Mathematics with Applications, Vol. 55, No. 10, 2008, pp. 2212-2226. http://dx.doi.org/10.1016/j.camwa.2007.11.012

[19] S. Shen, F. Liu and V. Anh, "Numerical Approximations and Solution Techniques for the Space-Time Riesz-Caputo Fractional Advection-Diffusion Equation," Numerical Algorithms, Vol. 56, No. 3, 2011, pp. 383-403. http://dx.doi.org/10.1007/s11075-010-9393-x

[20] C. Canuto, M. Y. Hussaini, A. Quarteroni and T. A. Zang,
"Spectral Methods. Fundamentals in Single Domains," Springer-Verlag, Berlin, 2006.

[21] D. Funaro and D. Gottlieb, “A New Method of Imposing Boundary Conditions in Pseudospectral Approximations of Hyperbolic Equations," Mathematical and Computer, Vol. 51, No. 184, 1988, pp. 599-613. http://dx.doi.org/10.1090/S0025-5718-1988-0958637-X

[22] G.-Q. Chen, Q. Du and E. Tadmor, "Spectral Viscosity Approximations to Multidimensional Scalar Conservation Laws," Mathematical and Computer, Vol. 61, No. 204, 1993, pp. 629-643. http://dx.doi.org/10.1090/S0025-5718-1993-1185240-3

[23] T. Y. Hou and R. Li, "Computing Nearly Singular Solutions Using Pseudo-Spectral Methods," Journal of Computational Physics, Vol. 226, No. 1, 2007, pp. 379-397. http://dx.doi.org/10.1016/j.jcp.2007.04.014

[24] S. Esmaeili and M. Shamsi, "A Pseudo-Spectral Scheme for the Approximate Solution of a Family of Fractional Differential Equations," Communications in Nonlinear Science \& Numerical Simulation, Vol. 16, No. 9, 2011, pp. 3646-3654. http://dx.doi.org/10.1016/j.cnsns.2010.12.008

[25] C. Li, F. Zeng and F. Liu, "Spectral Approximations to the Fractional Integral and Derivative," Fractional Calculus \& Applied Analysis, Vol. 15, No. 3, 2012, pp. 383406. http://dx.doi.org/10.2478/s13540-012-0028-x

[26] J. Shen and T. Tang, "Spectral and High-Order Methods with Applications," Science Press, Beijing, 2007.

[27] A. Quarteroni and A. Valli, "Numerical Approximation of Partial Differential Equations," Springer-Verlag, Berlin, 1997. 